\title{
Electromagnetic Compatibility Testing of Implantable Neurostimulators Exposed to Metal Detectors
}

\author{
Seth J. Seidman*, Wolfgang Kainz, Jon Casamento and Donald Witters
}

Food and Drug Administration (FDA), Center for Devices and Radiological Health (CDRH), 10903 New Hampshire Ave, Silver Spring, MD 20910, USA

\begin{abstract}
This paper presents results of electromagnetic compatibility (EMC) testing of three implantable neurostimulators exposed to the magnetic fields emitted from several walk-through and hand-held metal detectors. The motivation behind this testing comes from numerous adverse event reports involving active implantable medical devices (AIMDs) and security systems that have been received by the Food and Drug Administration (FDA). EMC testing was performed using three neurostimulators exposed to the emissions from 12 walk-through metal detectors (WTMDs) and 32 hand-held metal detectors (HHMDs). Emission measurements were performed on all HHMDs and WTMDs and summary data is presented. Results from the EMC testing indicate possible electromagnetic interference (EMI) between one of the neurostimulators and one WTMD and indicate that EMI between the three neurostimulators and HHMDs is unlikely. The results suggest that worst case situations for EMC testing are hard to predict and testing all major medical device modes and setting parameters are necessary to understand and characterize the EMC of AIMDs.
\end{abstract}

Keywords: EMC, EMI, metal detectors, implantable neurostimulators.

\section{INTRODUCTION}

This paper contains detailed information about the emissions from a wide range of metal detector systems and electromagnetic compatibility (EMC) test findings with three widely used implanted neurostimulators. The purpose of this paper is to inform physicians, neurostimulator manufacturers, and makers of metal detectors about possible EMC issues between implantable neurostimulators and metal detectors. It is also hoped that relevant guidance on this issue will be provided to neurostimulator patients by their physicians. Metal detectors referred to in this paper include both walk-through metal detectors (WTMDs) and hand-held metal detectors (HHMDs). Metal detectors typically use low frequency magnetic fields to detect concealed metal objects. A typical HHMD and WTMD can be seen in Figs. (1 and 2), respectively.

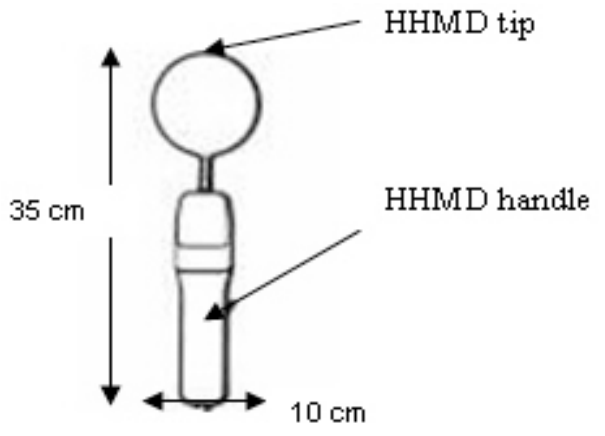

Fig. (1). Drawing of a typical HHMD with dimensions (thickness $3 \mathrm{~cm})$.

*Address correspondence to this author at the Food and Drug Administration (FDA), Center for Devices and Radiological Health (CDRH), 10903 New Hampshire Ave, Silver Spring, MD 20910, USA; Tel: 301-796-2477; Fax: 301-796-9927; E-mail: seth.seidman@fda.hhs.gov

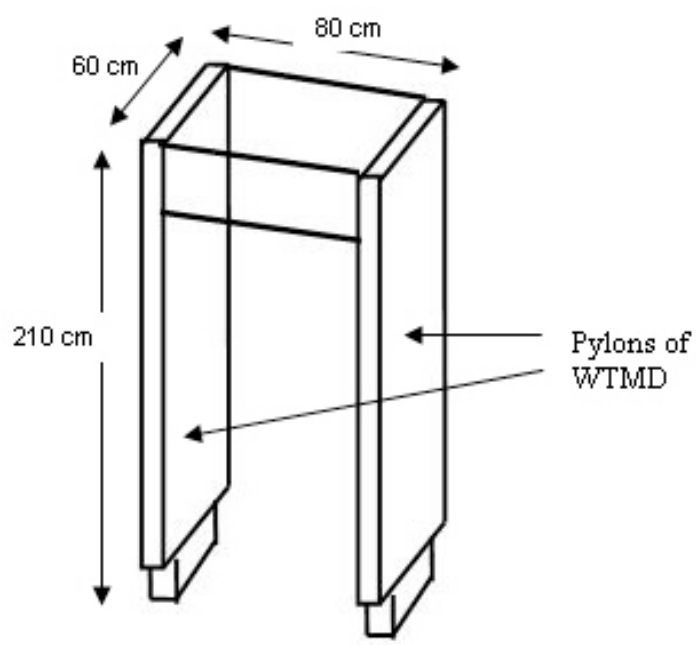

Fig. (2). Drawing of a typical WTMD with dimensions.

This work on medical device EMC continues because of the Food and Drug Administration's (FDA) concern of EMC issues between active implantable medical devices (AIMD) and security systems [1]. There have been 145 adverse event reports from 1987-2005 of AIMD malfunctions while in the vicinity of security systems. Figs. (3 and $\mathbf{4})$ shows the number of event reports related to EMI between AIMDs and security systems, categorized respectively by the type of AIMD and the type of security system. FDA's MAUDE (Manufacturer and User Facility Device Experience) data base is an important indicator of trends related to AIMD malfunctions, but the numbers reported are a clear underestimation. The increase in event reports since 2004 may be related to the increase in deployed security systems and the general rise in AIMD use. Previous analysis of these reports suggests creditable evidence of potentially significant 


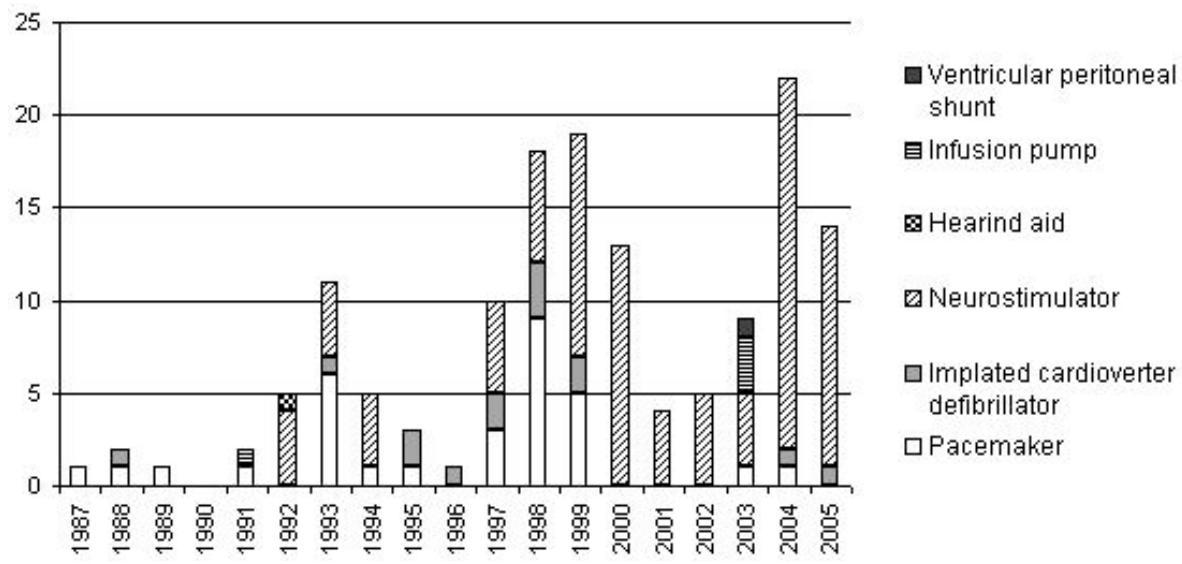

Fig. (3). Number of AIMD event reports between 1987 and 2005 linked to EMC issues with security systems, categorized by the type of AIMD.

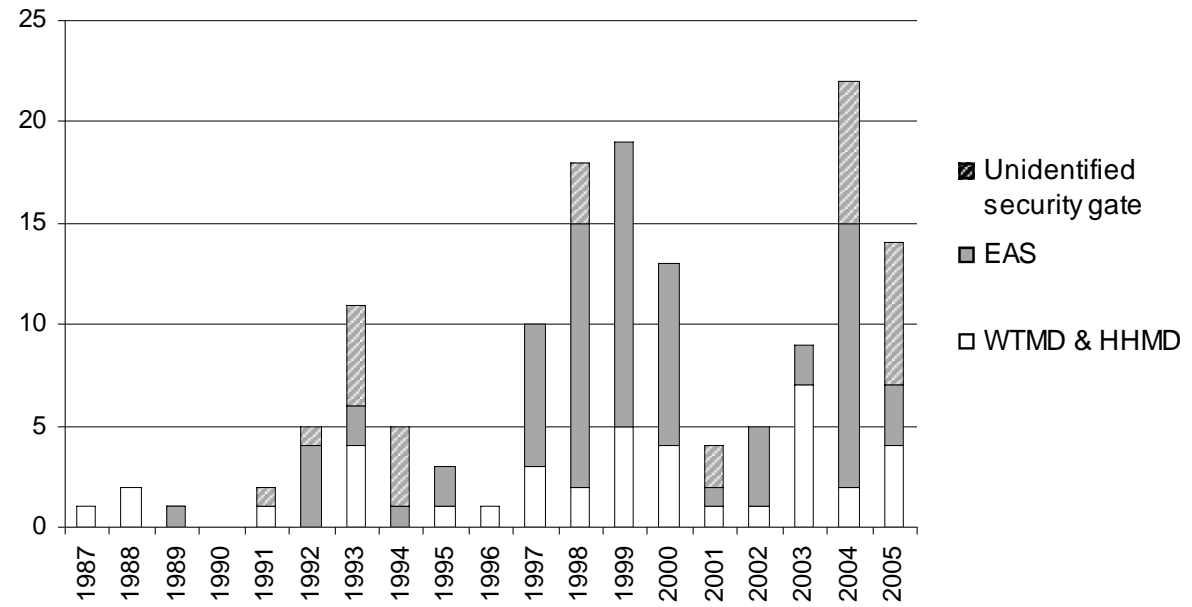

Fig. (4). Number of AIMD event reports between 1987 and 2005 linked to EMC issues with security systems, categorized by the type of security system.

electromagnetic interference (EMI) risks for patients using certain types of AIMDs [2]. Eisenberg [3] discusses one type of potentially harmful EMC event between a neurostimulator and electronic article surveillance (EAS) system. Metal detectors and EAS systems both emit RF energy in the form of low frequency magnetic fields.

Some EMC testing has been performed between neurostimulators and WTMDs and has been documented by Kainz [4]. However this testing involved a limited number of continuous wave (CW) WTMDs and one neurostimulator. Several other researchers have reported on EMI to nonneurostimulator AIMDs from security systems including work performed at the FDA on implantable cardiac pacemakers [5]. This work concluded that WTMDs with pulsed waveforms had the potential to cause EMI with implantable cardiac pacemakers. Extensive background information on neurostimulator EMC can be found in Hrdlicka [6] and Kainz [7].

\section{MATERIALS AND METHODOLOGY}

\section{A. Walk-Through Metal Detector Emissions}

Walk-through metal detector emissions were measured and evaluated as described by Kainz et al. [5]. Twelve
WTMDs from seven different manufacturers were evaluated. The waveform of each WTMD was digitally captured on a LeCroy Waverunner LT264 oscilloscope by placing a threeaxis magnetic field probe (model 1678.002) from Electric Research Management (ERM) within $5 \mathrm{~cm}$ of the transmitting pylon. The waveforms of the WTMD were categorized as either pulsed or continuous wave $(\mathrm{CW})$.

After the waveform was captured, a computer controlled three-axis scanning system [8] was used to scan seven planes (both horizontal and vertical) for each WTMD. Each WTMD's emissions were measured using the same threeaxis magnetic field probe from ERM. The results of the measurements were analyzed and the absolute maximum and two reasonable maximums were recorded for each WTMD. The absolute maximum field strength is the highest peak-topeak vector magnitude magnetic field measured. The reasonable maximum field strength is the highest peak-topeak magnetic field value within an area of the WTMD where an AIMD could be present while the patient is walking through. The boundary defining this area is more than $50 \mathrm{~cm}$ but less than $150 \mathrm{~cm}$ above the floor and not closer than $15 \mathrm{~cm}$ from either pylon. Two calculations were made using the reasonable maximum values within this area: 
1. Reasonable Maximum Normal to the Pylon: $H_{n}=H_{y}$

2

$$
\begin{aligned}
& \text { Reasonable Maximum Vector Magnitude: } \\
& H_{v}=\sqrt{H_{x}^{2}+H_{y}^{2}+H_{z}^{2}} .
\end{aligned}
$$

To find the likely exposure for the neurostimulator the two reasonable maximums were averaged over an $800 \mathrm{~cm}^{2}$ area in the shape of a square and defined as $\overline{H_{n}}$ and $\overline{H_{v}}$, respectively. $800 \mathrm{~cm}^{2}$ was the maximum loop area we could represent with our leads provided by Medtronic. Faraday's Law explains how current in a loop generates magnetic fields and why a larger loop size would generate a higher magnetic field (and thus more of a chance for EMI). Typical neurostimulator loop areas range widely from $10 \mathrm{~cm}^{2}$ to over $1000 \mathrm{~cm}^{2}$ [6]. An underestimation of the actual magnetic field produced by the WTMD is obtained by considering only one field component $\left(H_{n}\right)$ instead of all three $\left(H_{v}\right)$. An overestimation of the actual magnetic field produced by the WTMD is obtained by taking the vector magnitude $\left(H_{v}\right)$. Therefore the actual magnetic field exposure to the neurostimulator lies somewhere between the averaged values $\overline{H_{n}}$ and $\overline{H_{v}}$.

\section{B. Hand-held Metal Detector Emissions}

Thirty-two HHMDs were evaluated in a similar manner as the WTMD evaluations. The emission waveforms of the HHMDs were captured using a smaller version of the ERM three-axis magnetic field sensor calibrated for higher frequencies (model 1709.1) and the same digital oscilloscope as used to capture the WTMD emissions. The same threeaxis scanning system was used to map the magnetic field strengths from each HHMD. The emission measurements scanning protocol was different due to variation in size and geometry of a HHMD versus a WTMD. Five horizontal planes were scanned at $1.5,2.0,2.5,3.0$, and $5 \mathrm{~cm}$ above and parallel to the radiating surface of each HHMD. Two vertical planes, one aligned with the HHMD handle and one perpendicular to the HHMD handle were taken. No reasonable maximums were considered for HHMD measurements because the radiating surface is likely to be much closer to the AIMD than from a WTMD. The absolute maximum (i.e. the highest peak to peak vector magnitude magnetic field strength) was recorded for each HHMD.

\section{Neurostimulator EMC Testing with Walk-Through Metal Detectors}

It is cumbersome to test possible neurostimulator EMI directly in a WTMD due to the large testing volume of each WTMD. Therefore a metal detector simulator was designed in order to mimic magnetic fields emitted by each WTMD. The simulator consists of an arbitrary waveform generator, a trans-conductance amplifier, and a coil system. The simulator generates uniform magnetic fields in a volume 57 $\mathrm{cm}$ long, $42 \mathrm{~cm}$ wide, and $14 \mathrm{~cm}$ deep and is shown in Fig. (5). Details about the design are published in Misakian et al. [9] and simulator performance data are published in Kainz et al. [5]. All of the EMC testing performed for the WTMD emissions in the present study was performed using the simulator system.

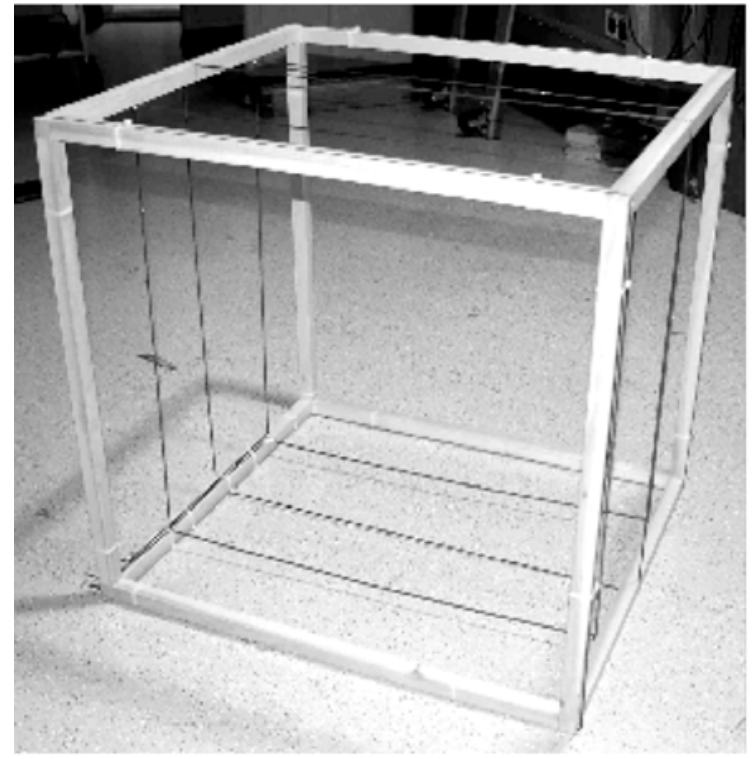

Fig. (5). Photo of the coil system. Five coil loops are mounted to a wooden frame to create a uniform exposure field where the neurostimulator could be tested.

Typical neurostimulator settings were chosen in accordance with Hrdlicka [6]. All neurostimulators tested had an amplitude of 3.0 Volts, a pulse width of 150 microseconds, and a pulse repetition rate of 70 Hertz. The neurostimulator leads formed a loop size of $800 \mathrm{~cm}^{2}$. The three neurostimulators tested along with their operating modes were:

- Medtronic Itrel3 (NS1): Quadripolar implantable pulse generator designed for spinal cord stimulation. Tested in both unipolar (NS1U) and bipolar (NS1B) modes of operation.

- Medtronic Soletra (NS2): Quadripolar neurostimulator designed for deep brain stimulation. Tested in both unipolar (NS2U) and bipolar (NS2B) modes of operation.

- $\quad$ Medtronic Synergy (NS3): Dual channel implantable pulse generator designed for spinal cord stimulation. Tested in SingleStim (NS3SS) mode of operation. DualStim mode allows each channel to have different amplitudes. DualStim mode settings were not tested. SingleStim and DualStim operate only in bipolar mode.

The neurostimulator output was monitored using the LeCroy Waverunner-2 LT-264 digital oscilloscope.

To perform testing in air (without a saline-filled phantom) the neurostimulator lead loop was closed using a $500 \mathrm{ohm}$ resistive load and connected to the oscilloscope using a twisted cable to avoid possible pick-up from the WTMD emissions. To perform testing in the saline-filled phantom the electrical path for the neurostimulator lead loop was closed with saline. In the saline-filled phantom the neurostimulator output was measured using the Srico Optical Voltage Probe and Receiver (Model 400-02M) by placing 
the two electrodes of the Srico probe in the saline bath near the tip of the neurostimulator lead. The neurostimulator can and lead configuration was placed on a flat plastic fixture and tied down with thin non-conducting strings. The plastic box used for saline testing (i.e., the saline-filled phantom) has dimensions of $42 \times 57 \times 30 \mathrm{~cm}$ and was filled with saline solution for a conductivity of $0.266 \mathrm{~S} / \mathrm{m}(0.14 \%$ salinity). This saline phantom test method is modified from the torso simulator method developed by Ruggera [10]. From previous testing [5] it is believed that air testing is a conservative alternative to testing in saline.

To observe possible neurostimulator interference the output signal of the neurostimulator was monitored on the oscilloscope. The field strength generated by the WTMD simulator started at $1 \mathrm{~A} / \mathrm{m}$ and was raised in $1 \mathrm{~A} / \mathrm{m}$ steps. Each field strength level was held for duration of 10 seconds. Interference was classified as a change in pulse shape of the neurostimulator output and was digitally captured on the oscilloscope, Fig. (6), along with the magnetic field strength.

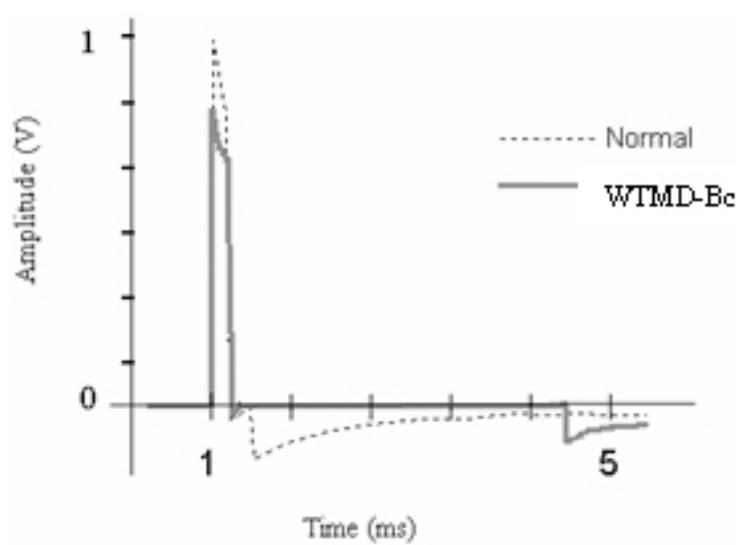

Fig. (6). Interference observed on a sample neurostimulator output signal. The dashed line represents a reference for the neurostimulator output signal with no metal detector emissions present. The solid line represents the neurostimulator output signal in the presence of emissions from a WTMD. This change in pulse area was classified as interference to a neurostimulator device.

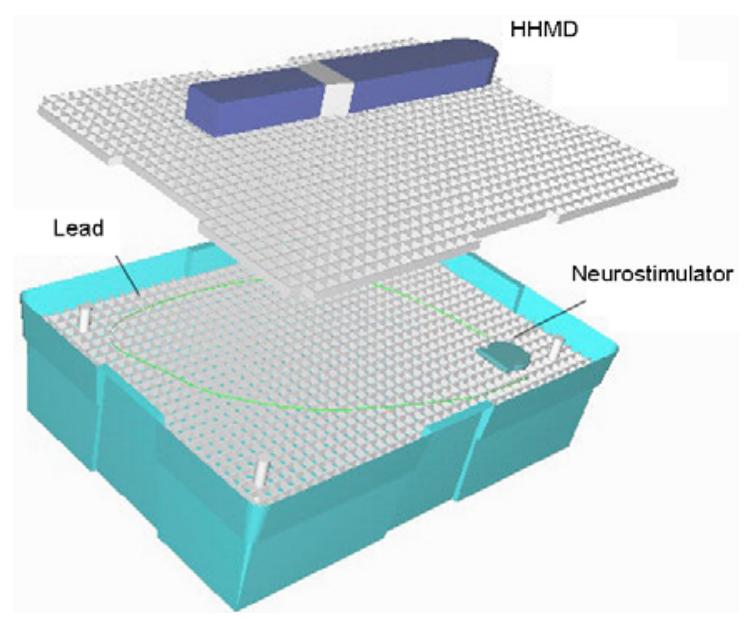

Fig. (7). Simulator configuration for EMC testing of a sample neurostimulator while exposed to HHMD emissions. The HHMD Support Grid was placed $1.5 \mathrm{~cm}$ above the neurostimulator.
This magnetic field strength level at which the device is affected is called the "interference threshold." Interference in an actual WTMD is possible if an interference threshold was found below $\overline{H_{n}}$. If an interference threshold is between $\overline{H_{n}}$ and $\overline{H_{v}}$ then it is not clear if the WTMD fields will interfere with the neurostimulator. If interference was found above $\overline{H_{v}}$ then that actual WTMD would not be able to produce such interference. The magnetic fields were measured using a Wandel and Goldermann EFA-2 EM Field Analyzer.

\section{Neurostimulator EMC Testing with Hand-Held Metal Detectors}

The three sample neurostimulator devices were exposed to the magnetic field emissions from 32 sample HHMDs to assess the EMC among these devices and the HHMDs. The actual HHMDs were used rather than the WTMD simulator because the WTMD simulator was not able to produce the emissions of all HHMDs. The same setup from the WTMD EMC tests was used to support and create a level plane to test the neurostimulators. An additional plastic fixture was placed over the neurostimulator plane to create a testing plane for the exposures. This plane created a $1.5 \mathrm{~cm}$ separation distance between the HHMD and neurostimulator as seen in Fig (7).

The active HHMD was then placed in one corner of the grid and moved in $3 \mathrm{~cm}$ intervals over the entire surface. The HHMD was held for a duration of 10 seconds in each location. Interference behavior was observed and recorded at each point. A quick sweep (1 second stop at each location instead of 10 seconds) was also performed with the HHMD rotated 90, 180, and 270 degrees, as well as with the tip touching the testing plane and the HHMD axis perpendicular to the testing surface (see Fig. (8)) to see if different orientations of the HHMD might result in interference of the neurostimulator.

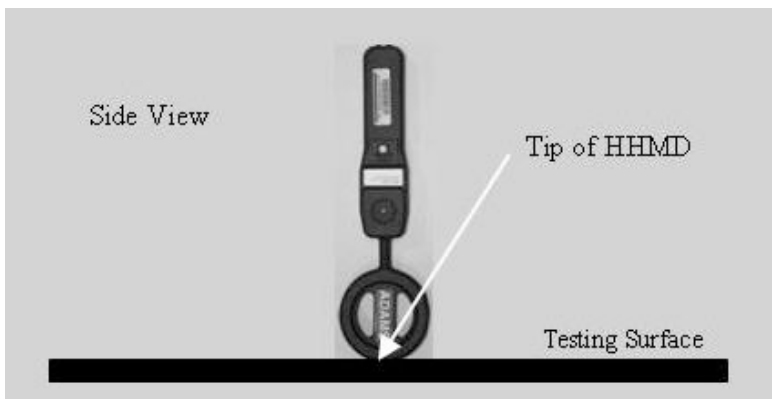

Fig. (8). Typical neurostimulator EMC testing with HHMDs occurred with the HHMD horizontal to the testing surface (not shown). Another orientation included the tip touching the testing plane and the HHMD axis perpendicular to the testing surface is shown above.

\section{RESULTS}

\section{A. Metal Detector Emission Data}

WTMDs were categorized as either pulsed or CW with frequencies ranging between $210 \mathrm{~Hz}$ and $7400 \mathrm{~Hz}$. The emission data for all 12 WTMDs is summarized in Table $\mathbf{1}$. 
Table 1. Summary of the Emission Measurements for 12 Different WTMDs. Primary Frequency, Waveform, Shortest Fall Time for Pulsed WTMD Signals. Max H Field Represents the Absolute Maximum Peak to Peak Vector Magnitude Recorded During Emission Measurements

\begin{tabular}{|c|c|c|c|c|c|c|}
\hline Manufacturer & Model & Abbreviation & Frequency $(\mathrm{Hz})$ & Waveform & Fall Time (us) & $\operatorname{Max} H$ Field (A/m) \\
\hline A & $\mathrm{a}$ & Aa & 276 & \multirow{9}{*}{ pulsed } & 25 & 163.0 \\
\hline \multirow{3}{*}{ B } & $\mathrm{a}$ & $\mathrm{Ba}$ & 400 & & 11.6 & 288.6 \\
\hline & $\mathrm{b}$ & $\mathrm{Bb}$ & 509 & & 10.4 & 226.4 \\
\hline & $\mathrm{c}$ & $\mathrm{Bc}$ & 240 & & 7.3 & 219.9 \\
\hline \multirow{4}{*}{$\mathrm{C}$} & \multirow{2}{*}{ a } & $\mathrm{Ca} 1$ & \multirow{2}{*}{569} & & \multirow{2}{*}{21.8} & \multirow{2}{*}{265.0} \\
\hline & & $\mathrm{Ca} 2$ & & & & \\
\hline & \multirow{2}{*}{$\mathrm{b}$} & $\mathrm{Cb} 1$ & \multirow{2}{*}{210} & & \multirow{2}{*}{47} & \multirow{2}{*}{473.6} \\
\hline & & $\mathrm{Cb} 2$ & & & & \\
\hline $\mathrm{D}$ & $\mathrm{a}$ & $\mathrm{Da}$ & 210 & & 43.5 & 378.1 \\
\hline \multirow{3}{*}{$\mathrm{E}$} & a & $\mathrm{Ea}$ & $3520,4690,5640$ & multiple CW & - & 122.6 \\
\hline & $\mathrm{b}$ & $\mathrm{Eb}$ & 4535 & \multirow{4}{*}{$\mathrm{CW}$} & - & 91.9 \\
\hline & $\mathrm{c}$ & Ec & 7400 & & - & 69.3 \\
\hline $\mathrm{F}$ & $\mathrm{a}$ & $\mathrm{Fa}$ & 269 & & - & 465.2 \\
\hline G & a & $\mathrm{Ga}$ & 5170 & & - & 18.8 \\
\hline
\end{tabular}

For the measurements reported in this paper the difference between $\overline{H_{n}}$ and $\overline{H_{v}}$ averaged about $11 \%$ with a maximum of $22 \%$.

Emission maps from a sample HHMD is shown in Figs. (9-11). All except one HHMD emitted CW waveforms with frequencies ranging from $10 \mathrm{kHz}$ to $1856 \mathrm{kHz}$. The pulsed waveform that HHMD-M emitted was unique among the samples. The maximum field strengths emitted by the other HHMDs ranged from 2.1 to $67.3 \mathrm{~A} / \mathrm{m}$, with HHMD-M emitting significantly higher magnetic field strengths of over $600 \mathrm{~A} / \mathrm{m}$. The high magnetic field emissions from HHMD-M exceeded the range of the 1709.1 ERM probe at $2.5 \mathrm{~cm}$ from the surface. The emissions were then measured using an 1850.002 ERM probe at $2 \mathrm{~cm}$ measuring $600 \mathrm{~A} / \mathrm{m}$. The emission data for all HHMDs is summarized in Table 2.

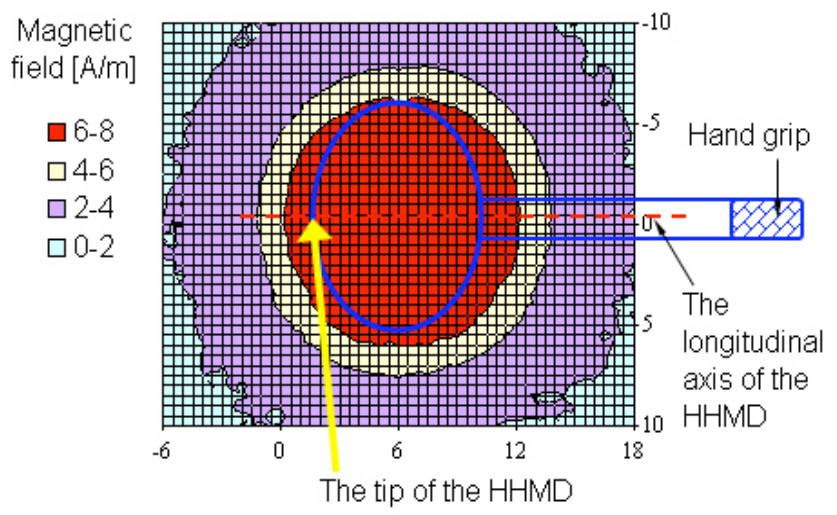

Fig. (9). HHMD emissions from a horizontal plane (overhead view). Axes coordinates in $\mathrm{cm}$.

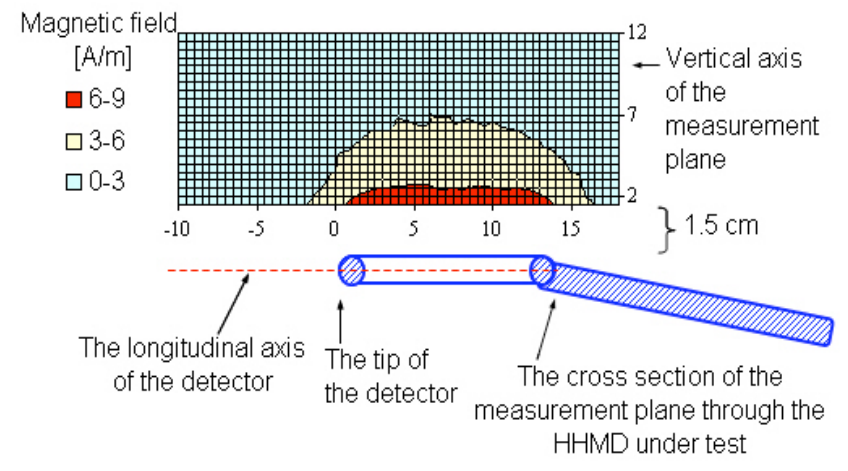

Fig. (10). HHMD emissions from a vertical-handle-aligned plane (side view). Axes coordinates in $\mathrm{cm}$.

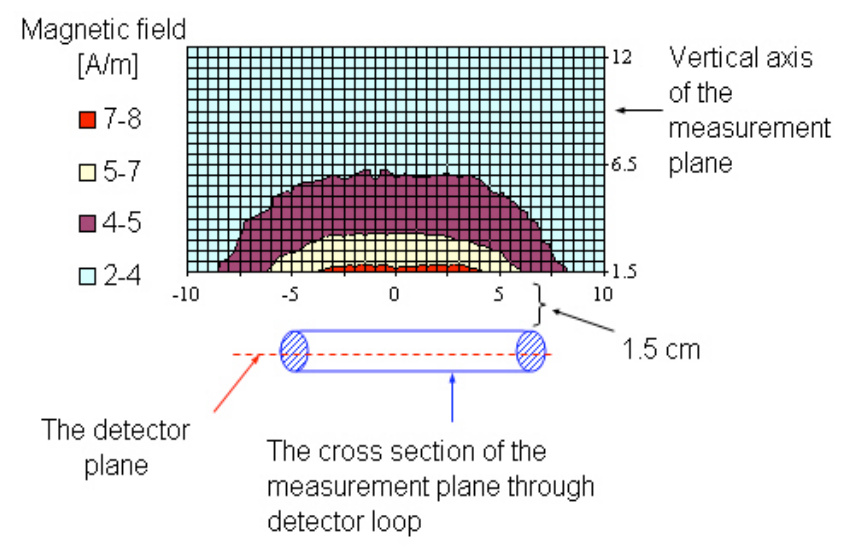

Fig. (11). Example of HHMD emissions from a vertical-handlenormal plane (front view). Axes coordinates in $\mathrm{cm}$. 
Table 2. Summary of the Emission Measurements for 32 HHMDs. Max H Field Represents the Absolute Maximum Peak to Peak Vector Magnitude Recorded During Emission Measurements

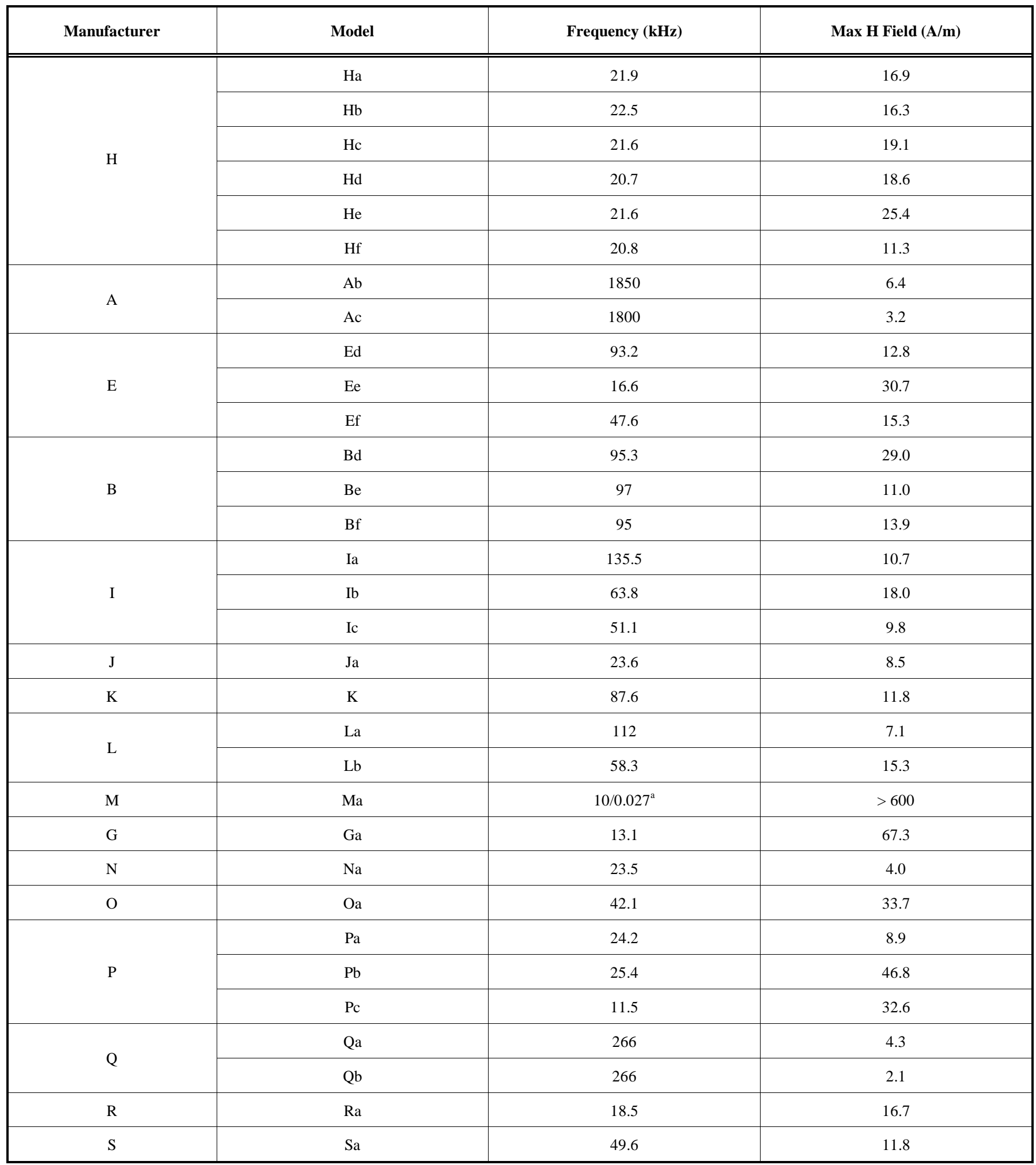

*Pulsed Waveform, Duration 50 us, Repetition Freq $26.75 \mathrm{~Hz}$.

\section{B. Walk-Through Metal Detectors}

Findings for NS1B during air tests indicated the output of the device was affected for exposure levels below $\overline{H_{n}}$ from WTMD-Bc2. During saline testing the interference threshold for NS1U was below $\overline{H_{n}}$ and for NS1B the interference threshold was below $\overline{H_{v}}$ from WTMD-Bc2. In most cases the interference behavior of NS1 in saline was similar to the behavior in air. Saline was generally the worst case scenario 


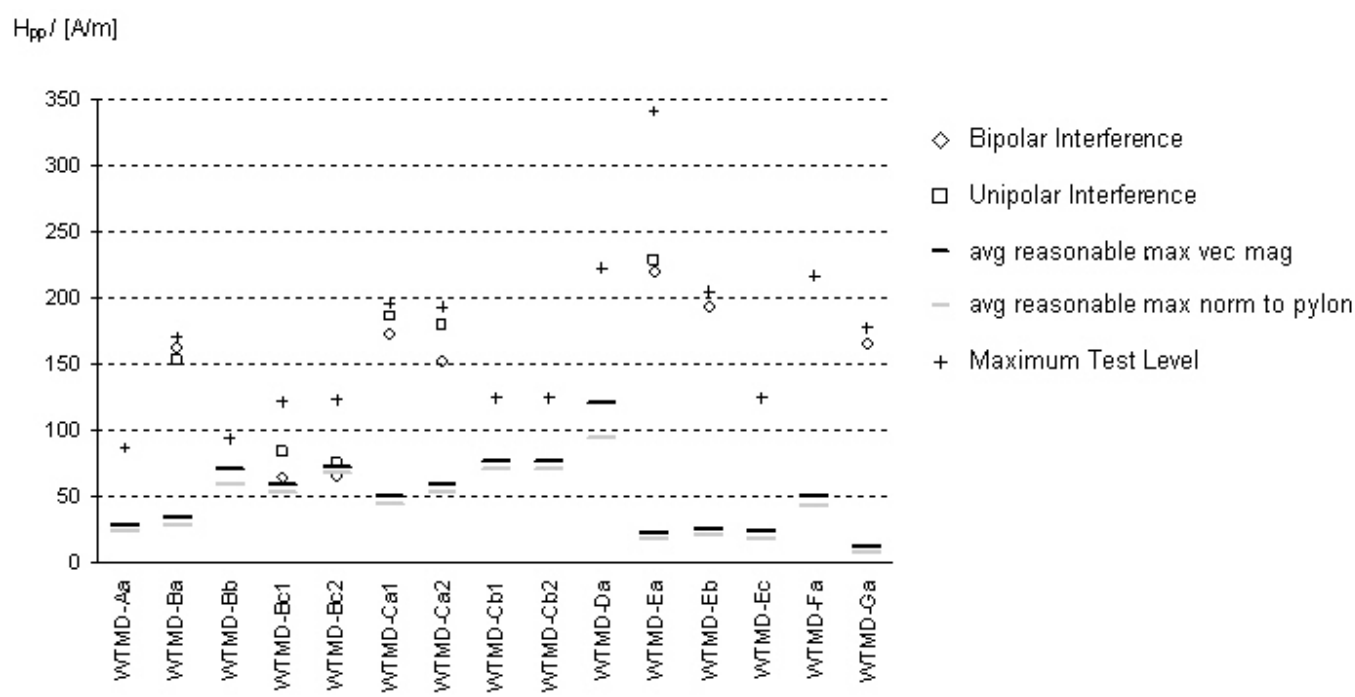

Fig. (12). Interference thresholds for testing in air of the NS1 (Itrel 3) neurostimulator. Interference is possible in the actual WTMD when below the averaged reasonable maximum vector magnitude $\left(\overline{H_{v}}\right)$. The magnetic fields are given as peak-to-peak values in A/m. Missing interference symbols for a particular WTMD signal indicates that no interference was observed up to the maximum test level for that WTMD signal.

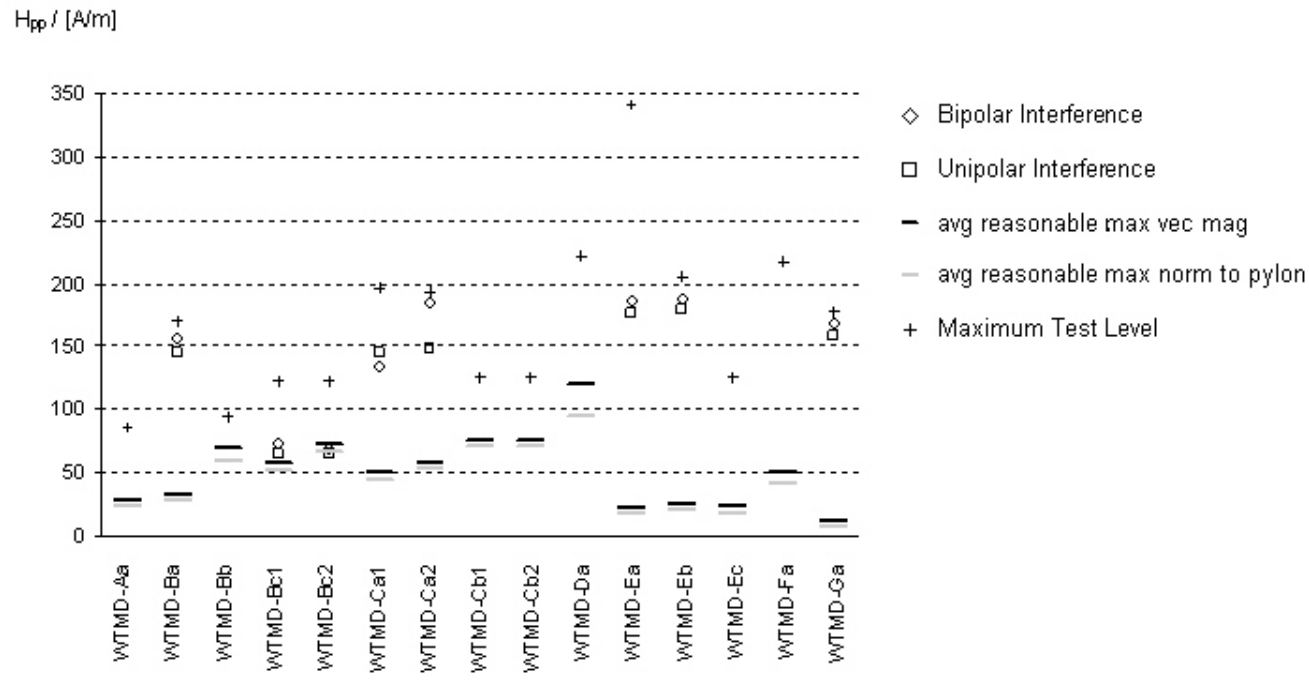

Fig. (13). Interference thresholds for testing in saline of the NS1 (Itrel3) neurostimulator. Interference is possible in the actual WTMD when below the averaged reasonable maximum vector magnitude $\left(\overline{H_{v}}\right)$. The magnetic fields are given as peak-to-peak values in $\mathrm{A} / \mathrm{m}$. Missing interference symbols for a particular WTMD signal indicates that no interference was observed up to the maximum test level for that WTMD signal.

as interference thresholds were lower than testing in air. There were also several scenarios where interference could be found in saline and not in air. All interference thresholds for NS1 from testing in air and saline can be found in Figs. (12 and 13), respectively.

No interference up to the maximum test level (see Figs. (12-13) for maximum test levels) was found testing NS2U, NS2B, or NS3 for both pulsed and CW type WTMD signals.

\section{Hand-Held Metal Detectors}

No EMI behavior was observed for all three neurostimulators in all operating modes in both air and saline while exposed to any HHMD. However, a superimposed signal of HHMD-M was picked up by the neurostimulator leads when NS1 was exposed to HHMD-M (see Fig. (14)). It is unknown what type of effect the patient could receive, if anything, from such an exposure.

\section{DISCUSSION AND CONCLUSION}

EMC testing was performed for three sample neurostimulators in all operating modes exposed to 12 WTMDs and 32 HHMDs for both air and saline test methods (totaling 470 testing combinations). From the testing results using the actual HHMDs it seems that neurostimulator interference from a HHMD is unlikely. This can be explained because the average magnetic field over the 
neurostimulator loop area is much less from HHMDs than from WTMDs.

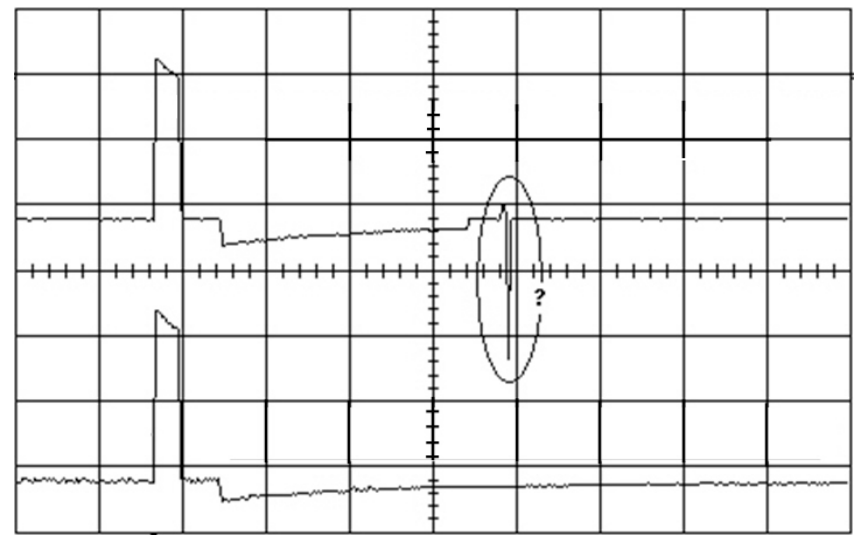

Fig. (14). The top signal is the NS1U output signal when exposed to emissions from HHMD-M. The circle shows a superimposed signal of HHMD-M that was picked up by the neurostimulator leads when NS1 was exposed to HHMD-M. The bottom signal shows the NS1U reference output signal with no metal detector emissions present.

Interference was observed for exposures below $H_{n}$ in two of these combinations with WTMDs. The two combinations occurred with the NS1 neurostimulator and WTMD-Bc2. The results as a whole do not prove the hypothesis [5] that pulsed waveforms are more likely to cause interference than $\mathrm{CW}$ waveforms. However, the two observed cases of interference on the device output did come from a pulsed WTMD, and so it seems to provide further support for this hypothesis. Discussions with neurostimulator manufacturers suggest that they are aware of possible reactions to security system emissions and newer generation devices have been re-designed and tested for greater immunity. Hrdlicka [6] states that it is possible that "walkthrough devices may induce significant voltage in the lead system to cause stimulation or occasional shocking." Manufacturers of neurostimulators believe the effect of unintentional shocking could be caused by a misbalance of positive and negative charges as a consequence of a disturbed pulse (personal communication between FDA and neurostimulator manufacturer). Manufacturers also suggest that interference, such as seen in Fig. (9), can be eliminated by turning the neurostimulator off before being exposed to a metal detector. However certain patients may not want to turn their neurostimulators off because this could lead to a sudden return of symptoms.
An analysis of the interference levels from NS1 show that testing in air is not always the worst case situation as was seen for implanted cardiac pacemaker interference [5]. The present testing also showed that in some cases unipolar lead configuration was not always the worst case situation. In terms of EMC and potential EMI for medical devices, worst case situations are very difficult to predict. For this reason, it is encouraged that medical device testing be performed for all major device setting parameters and lead configurations. EMC testing should be performed up to, and beyond, the emission levels of today's security systems in order to address the potential risks for EMI. The number of metal detectors will increase over the next years and AIMDs should be able to operate without interference while exposed to them. FDA is working with standardization committees and industry to develop standardized EMC test methods for neurostimulators and other AIMDs.

\section{REFERENCES}

[1] "Important Information on Anti-Theft and Metal Detector Systems and Pacemakers, ICDs, and Spinal Cord Stimulators", FDA Center for Devices and Radiological Health Letter to Cardiologists, Cardiac Surgeons, Neurosurgeons, and Emergency Physicians, September 28, 1998.

[2] D. Witters, S. Portnoy, J. Casamento, P. Ruggera, and H. Bassen, "Medical Device EMI: FDA Analysis of Incident Reports, and Recent Concerns for Security Systems and Wireless Medical Telemetry," in IEEE EMC International Symposium, pp. 12891291, 2001.

[3] E. Eisenberg, and H. Waisbrod, "Spinal cord stimulator activation by an antitheft device," J. Neurosurg., vol. 87, pp. 961-962, Dec. 1997.

[4] W. Kainz, and G. Neubauer, "Interference of electronic article surveillance systems and metal detector gates with implantable neurological pulse generators," in EBEA 2001, 5th International Congress of the European Bio-Electromagnetics Association (EBEA), 2001.

[5] W. Kainz, J. Casamento, P. Ruggera, D. Chan, and D. Witters, "Implantable Cardiac Pacemaker EMC Testing in a Novel Security System Simulator," IEEE Trans. Biomed. Eng., vol. 52, no. 3, pp. 520-530, March 2005.

[6] G. Hrdlicka, "Implantable Neurostimulators and the EMC Environment," in Electromagnetic Compatibility, 2002 IEEE International Symposium, 2002.

[7] W. Kainz, G. Neubauer, S. Alesch, and O. Jahn, "Electromagnetic compatibility of electronic implants - review of the literature," Middle Eur. J. Med., vol. 113, pp. 903-914, 2001.

[8] J. Casamento, "Characterizing electromagnetic fields of common electronic article surveillance systems," Compliance Eng., vol. 16 , pp. 42-52, Sept./Oct. 1999.

[9] M. Misakian, J. Casamento, and O. Laug, "Development of Emulator for Walk-Through Metal Detectors," IEEE Trans. Electromagn. Compatibil., vol. 44, no. 3, pp. 486-489, Aug. 2002.

[10] P. Ruggera, D. Witters, and H. Bassen, "In vitro Testing of Pacemakers for Digital Cellular Phone Electromagnetic Interference," Biomed. Instrum. Technol., vol. 31, pp. 358-371, July/August 1997. 